\title{
Homocystine as an Important Prognostic Marker in Neurodegenerative Diseases
}

\author{
Dr. Md. Raknuzzaman ${ }^{1 *}$, Tasnim Jannaty ${ }^{2}$, Dr. Md. Masud Rana ${ }^{3}$, Abu Shams Md. Hasan Ali Masum ${ }^{4}$ Dr. Md. Shofikul
} Islam $^{5}$, Dr. Anis Ahmed ${ }^{6}$

\footnotetext{
${ }^{1}$ Junior Consultant, National Institute of Neurosciences \& Hospital, Dhaka, Bangladesh

${ }^{2}$ Department of Biochemistry \& Molecular Biology, Dhaka University, Dhaka, Bangladesh

${ }^{3}$ Assistant Professor, Department of Neurology, Bangabandhu Sheikh Mujib Medical University, Dhaka, Bangladesh

${ }^{4}$ Assistant Professor, Department of Neurology, Anwer Khan Modern Medical College, Dhaka, Bangladesh

${ }^{5}$ Resident, Department of Neurology, Bangabandhu Sheikh Mujib Medical University, Dhaka, Bangladesh

${ }^{6}$ Assistant Professor, Department of Neurology, Bangabandhu Sheikh Mujib Medical University, Dhaka, Bangladesh
}

DOI: $10.36347 /$ sjams.2020.v08i05.032

| Received: 27.04.2020 | Accepted: 04.05.2020 | Published: 27.05.2020

*Corresponding author: Dr. Md. Raknuzzaman

Abstract

Original Research Article

Background: Neurodegeneration is the progressive loss of structure or function of neurons, including the death of neurons. The major neurodegenerative diseases (ND) are Alzheimer's disease (AD), Parkinson's disease, Amyotrophic lateral sclerosis (ALS), Huntington's disease, Prion diseases, Multiple sclerosis (MS), Neuronal cell death \& Epilepsy. These occur as a result of neurodegenerative processes. Homocysteine may promote Alzheimer's disease by more than one mechanism, including oxidative stress, neuronal cell damage, tau phosphorylation, enhancement of beta-amyloid aggregation, and hyperactivation of NMDA (N-methyl-D-aspartate) receptor. The diseases are incurable of progressive degeneration and/or death of neuron cells. On the other hand, homocysteine is a non-proteinogenic $\alpha$ amino acid. Serum homocysteine level is considered as a prognostic marker in neurodegenerative diseases. Objective: The aim of this study was to evaluate the serum homocysteine level as an important prognostic marker in neurodegenerative diseases. Methodology and Materials: This case-control study was conducted at neuromuscular disorder clinic, inpatient and outpatient department of neurology, BSMMU Dhaka, Bangladesh during the period from April-2018 to September-2019. Non-random purposive sampling technique was followed to collect the sample. In total 42 patients of several types of neurodegenerative diseases. Among them Alzheimer's disease patients were 15(36.5\%), Parkinson's disease 11(24.5\%), Amyotrophic lateral sclerosis (ALS) disease were 8(18\%), Huntington's disease were $6(15 \%)$ and Prion disease were 2(5\%) were finalized as case and 42 people without any neurodegenerative disease were finalized as the control group. At the end of data collection, the mean and standard deviation of serum levels of Homocysteine of both case and control group were calculated and analyzed. Results: The correlation coefficient (r) of $\Delta \mathrm{FS}$ with Hcy is +.142 ; which indicates Hcy is positively correlated with progression rate $\Delta \mathrm{FS}$, and the correlation is also weak. So there is increasing Hcy level with the increase of progression rate $\triangle F S$. BMI positively correlated with serum Hcy and age negatively correlated with Hcy. Conclusion: In this study in comparing the serum Homocysteine between two groups unpaired student t-test was done. Here $\mathrm{p}$-value was found $0.003(\mathrm{p}<0.05)$ which indicated was a significant correlation between the groups. So Homocysteine may be considered as a prognostic marker in neurodegenerative diseases. But it may not be considered as a very potential prognostic marker.

Keywords: Homocystine, Prognostic marker, Neurodegenerative diseases.

Copyright @ 2020: This is an open-access article distributed under the terms of the Creative Commons Attribution license which permits unrestricted use, distribution, and reproduction in any medium for non-commercial use (NonCommercial, or CC-BY-NC) provided the original author and source are credited.

\section{INTRODUCTION}

Homocysteine (Hcy) is the demethylated derivative of methionine, which after conversion to Sadenosyl methionine, is the most important methyl group donor in the body. Excess of Homocysteine (Hcy) is considered a neurotoxin since it has a very deleterious effect on the nervous system. It is a sulfurcontaining amino acid that is reversibly formed and secreted during metabolism. Preclinically and clinically,
Hcy exhibits several neurological mechanisms that have been reported in the pathogenesis of Alzheimer's disease, stroke, Parkinson's disease, Amyotrophic Lateral Sclerosis (ALS) disease, Huntington's disease, Prion, Multiple Sclerosis (MS), Stroke, Neuronal cell death, and Epilepsy. Homocysteine may endorse Alzheimer's disease by more than one mechanism, including oxidative strain, neuronal cell injury, tau phosphorylation, enhancement of beta-amyloid 
aggregation, and hyperactivationof NMDA (N-methylD-aspartate) receptor. Moreover, it increases the production of chemokines by stimulation of nuclear factor-kappa B. It is well known that the use of levodopa diminishes the symptoms of Parkinson's disease but also leads to an elevation in the level of homocysteine. In this review, by discussing its neurodegenerative effects we highlight the associate relationship between hyperhomocysteinemia and neurological disorders. Homocysteine is converted back into methionine with the help of betainehomocysteinemethyltransferase or methionine synthase and cobalamin (remethylation pathway). Homocysteine transformed into cystathionine by the action of cystathionine beta-synthase (CBS) with pyridoxine performing as co-factor. Homocysteine acts as a precursor and metabolite to S-adenosyl methionine (AdoMet) and S-adenosylhomocysteine (AdoHcy) respectively [1]. The ratio of these 2 is referred to as methylation potential (MP) [2]. Low folate and increased Hcy concentrations in blood are associated with poor cognitive performance in the general population. Elevated levels of Hcy result in neurotoxic and vast toxic effects in Alzheimer's disease dementia disease, suggesting that Hcy is a direct marker of early cognitive decline. ${ }^{3}$ However, the pathogenesis of Hcy is still not clear in vascular dementia. The levels of serum folate, vitamin B12, and plasma Hcy were studied in vascular Alzheimer's and dementia disease. It was found that Hcy was increased, while folate and vitamin B12 were decreased in both of these diseases. This suggests that supplementation of folic acid and vitamin B12 could be of benefit in Alzheimer's disease and dementia [4]. A randomized, double-blind, placebocontrolled study was conducted in the Netherlands on 818 subjects, men and post-menopausal women aged 50-70 years who used placebo or $800 \mu \mathrm{g} /$ day folic acid for 3 years. Serum folate concentrations increased and plasma Hcy concentrations decreased in participants taking folic acid compared with those taking placebo. Alzheimer's disease is a neurodegenerative disease, which results from the deposition of amyloid-beta (Ab) peptides and a form of tau protein. The primary causes of this multifactorial disease are the accumulation of A $\beta 5$-x, a peptide cleaved from the amyloid precursor protein (APP) by the stepwise enzymatic action of the beta-secretase (BACE-1) and gamma-secretase [5]. Alzheimer's disease is the most common type of dementia and accounts for $50-80 \%$ of dementia cases. Currently, as of 2013, more than 44.4 million people in the world suffer from dementia and this number is expected to increase to over 115 million by 2050 . According to statistics of AD (www.Alz.co.uk /research /statics), the fastest growth in the elderly population is taking place in the north and south Asia, as well as in the countries of the western pacific region. AD requires long-term care entailing a considerable financial burden on the families affected as well as on the society as a whole. Scientists working in this area of research are coming up with new strategies and treatments for the prevention and progression of the disease. Moderate hyperhomocysteinemia (Hhcy) is considered to be one of the modifiable risk factors for AD. Regland was the first one to report the elevated level of Hhcy in primary degenerative dementia, and after that, several studies have been carried out to confirm that the elevated level of Hcy is associated with AD [6]. Damaging of dopamine-producing cells would result in a neurodegenerative disease called Parkinson's disease (PD), characterized by the loss of motor control. About $10-30 \%$ of patients with PD have shown evidence of Hhcy [7]. Examples of both the cause and effects of high levels of Hcy are evident in patients with PD. In 2010, Levin et al. found a significantly high level of Hcy and methyl-malonate in patients suffering from distinct neurodegenerative disease, progressive supranuclear palsy (PSP), ALS and PD [8]. A numerous studies showed Hhcy in patients with PD is associated with L-dopa (LD) treatment and is not found to be associated with non-demented older patients with PD [9]. Huntington's disease (HD) is an autosomal dominant neurodegenerative disease with CAG repeat at the 50 ends of the gene. In the normal population, there are about 8-39 repeats of CAG, but patients with HD have about 36-121 repeats of CAG associated with the earlier onset of HD with symptoms of movement disorder, cognition impairment and variable psychiatric symptoms [10]. Huntington protein has a high affinity to bind with the CBS enzyme and thereby inhibited its activity which in turn increased the level of Hcy. In an HD patient, Hhcy has been observed as the mutated Huntington protein influences the Hcy metabolism by modulating the cystathionine beta-synthase activity [11]. However, experimental data showed-even a modest increase of homocysteine may induce oxidative stress and DNA damage and support the hypothesis that oxidative stress, which is increased by homocysteine accumulation, might playing a role in ALS pathogenesis [12]. Neurodegenerative diseases characterize a major threat to human health. These age-dependent disorders are becoming increasingly prevalent, in part because the elderly population has increased in recent years [13]. Examples of neurodegenerative diseases are Alzheimer's disease, Parkinson's disease, Huntington's disease, Amyotrophic lateral sclerosis, front temporal dementia, and the spin cerebellar ataxias. These diseases are diverse in their pathophysiology-with some causing memory and cognitive impairments and others affecting a person's ability to move, speak and breathe [14]. In a systematic review, the mean age of ALS onset was 62 years and the incidence and mortality rates of ALS have been slowly increasing over the decades [15]. It is now considered as the 3rd most common neurodegenerative disorder after AD \& PD [16]. Prion diseases of humans which also known as transmissible spongiform encephalopathies (TSEs) are fatal neurodegenerative disorders, which comprise Kuru, Creutzfeldt-Jakob disease (CJD), Gerstmann-SträusslerScheinker syndrome (GSS), and fatal familial insomnia (FFI). The various subtypes of ND diseases have 
different characteristics activated onset durations of the disease, genetic histories of the patients, clinical indexes, neuropathology, and scrapie-like prion protein (PrPSc) molecular [17, 18]. Alzheimer's disease is characterized by heart loss or mild cognitive impairments (MCI) of normal age with early dementia. Patients of MCI can perform their routine activities smoothly but with some noticeable memory fluctuations or another sought of cognitive disturbances. MCI might be amnestic and non-amnestic but both of them fail to be diagnosed for dementia. MCI might be marked as an outset of dementia thanks to the very fact that patients with MCI are found more susceptible to dementia than normal cognitive patients. Clinical data reports recommend that hyperhomocysteinemia may be selfdetermining risks think about transmuting a healthy cognitive person to dementia in both normal elderly persons also as persons affected by Alzheimer's disease [17]. Hyperhomocysteinemia may be a condition developed in healthy individuals with or without MCI. A co-relation builds up between learning and hippocampal function and hyperhomocysteinemia suggests that hyperhomocysteinemia degrades cognitive functions in both controls healthy person and MCI patients by triggering brain atrophy with MCI [19]. Another study shows a link between homocysteine, hippocampal plasticity, and synaptic transmission indicating learning and memory shortcomings [20]. The neurotoxicity caused by homocysteine might be explained by the auto-oxidation of homocysteine that indications to the formation of reactive oxygen species which becomes the rationale for neuroinflammation and apoptosis [21]. Hyperhomocysteinemia is stated to switch the structure and performance of cerebral blood vessels by oxidative strain and endothelial dysfunctions that cause perfusion impairment followed by neuronal disturbances and marked to be as risk factors within the pathogenesis of vascular dementia and Alzheimer's disease [22]. The relation between hyperhomocysteinemia and dementia has gathered tons of interest in determining the mechanism related to it. It's believed that it acts by being an excitatory neurotransmitter that competes with inhibitory neurotransmitter gamma-aminobutyric acid (GABA). It obstructs GABA-A/B receptors by inducing micro vascular permeability which then grows redox strain, which further activates disinterring and metalloproteinase, terminating tissue inhibitors of metalloproteinase. These disturb the barrier matrix resulting in vascular dementia [23]. Zhang et al., concluded that rises in plasma homocysteine levels possibly induce acquisition of amyloid-beta peptides and also increase Alzheimer's like tau phosphorylation within the rat. It had been also found that homocysteine made neurons susceptible to amyloid-beta toxicity and in hippocampal neurons, it tarnishes the method of DNA repair [24]. Thus the study shownthat serum homocysteine level as a crucial prognostic marker in Alzheimer's and other major neurodegenerative diseases.

\section{ОВJECTIVES \\ General Objective}

- To evaluate the serum Homocystine level as an important prognostic marker in neurodegenerative diseases.

\section{Specific Objective}

- To see the relation between duration of neurodegenerative diseases and serum homocysteine level.

\section{Methodology \& Materials}

This case-control study was conducted at neuromuscular disorder clinic, inpatient and outpatient department of neurology, BSMMU Dhaka, Bangladesh during the period from April- 2018 to September 2019. Non-random purposive sampling technique was followed to collect the sample. In total 42 patients of several types of neurodegenerative diseases (ND) were finalized as case and 42 people without any neurodegenerative disease were finalized as the control group. All subjects were selected according to the revised El Escorial criteria and fulfilled the criteria for possible, probable, probable-laboratory-supported or definite neurodegenerative disease. None of the patients had a family history of neurodegenerative disease. The severity of the disorder was measured. Otherwise healthy individuals of well-matched controls for each patient in terms of age and sex were taken. Both groups of the patient were selected from neuromuscular junction disorder clinic, Outpatient and inpatient department of neurology of BSMMU. Informed written consent was taken from each patient. After taking a proper history, physical and neurological examination, NCS and EMG (for cases only, done in our electrophysiology $\mathrm{Lab}$ ) fasting serum homocysteine level and other relevant investigations were done. Proper diagnosis and treatment were ensured for each person of both groups. For the serum homocysteine level, $4 \mathrm{ccs}$ of the whole blood sample were collected after 8 hours of fasting. The blood sample was collected from the antecubital vein after asepsis with $0.5 \%$ chlorhexidinegluconate. Freshly drawn blood by standard venipuncture procedure and was collected into plastic screw-capped vial labeled with the patient's identification number and was kept on ice water and serum was harvested within 30 minutes of drawing. For harvesting serum, whole blood was centrifuged for 10 minutes at 4,000 rpm. Then an appropriate amount of serum was dispensed into plastic screw-capped vial labeled with the patient's identification number. The serum homocysteine level was estimated by using an automated analyzer architect plus ci4100 as soon as possible. The normal range of the serum homocysteine level was based on the reference used for serum homocysteine level in the laboratory of the department of biochemistry \& molecular biology, BSMMU, Dhaka, Bangladesh. All other necessary biochemical investigations of all patients were performed in the 
laboratory of the department of biochemistry \& molecular biology, BSMMU, Dhaka, Bangladesh. After completion of data collection, data were analyzed with SPSS (version 23.0) software properly. At the end of data collection, the mean and standard deviation of serum levels of homocysteine of both case and control group were calculated. Statistical significance was set at $\mathrm{p}<0.05$ for all of the data analyses.

\section{RESULTS}

In this study, the respondents were taken from all age groups irrespective of sex and religion. The mean age of the cases was $41.55 \pm 14.9$ years and the mean age of the control was $42.78 \pm 14.87$ years which were quite next to each other. There was no significant difference in age distribution between case and control, $(p>0.05)$. The minimum age of respondents was 16 years and the maximum age of 78 years. Maximum respondents were between 30-50 years of age group. Unpaired t-test was done and the p-value was not significant. Out of 42 cases, $33(78.6 \%)$ were male and $9(21.4 \%)$ were female. Among the control population, $33(78.6 \%)$ were male and $9(21.4 \%)$ were female. Chisquare test was done and the p-value was not significant. The respondents were measured by the BMI (categorized in body build). The mean BMI of the respondents (cases) was 20.26 with SD (2.11) and the mean BMI of controls was 21.35 with SD (2.10). The majority of them were healthy levels of BMI that are cases 35 and controls 38. But it is observed that underweight (BMI) is more likely cases group (7). Besides these, in diseases patterns among case group, it was found that the highest $36.5 \%(\mathrm{n}=15)$ patients were with Alzheimer's disease whereas 24.5\% $(n=11)$ were with Parkinson's disease, $19 \%(\mathrm{n}=8)$ were with Amyotrophic lateral sclerosis (ALS) disease, 15\% ( $n=6)$ were with Huntington's disease and $5 \%(\mathrm{n}=2)$ were with prion disease. Unpaired t-test was done p-value was not significant. Duration of the disease state of the neurodegenerative diseases patients was categorized to different groups starting from less than 3 months to more than 12 months and had disease duration; mean at the time of examination was $14.34 \pm 11.23$ months. Study variable analysis revealed that the mean of homocysteine in neurodegenerative diseases cases is higher $(14.06 \mu \mathrm{mol} / \mathrm{L}$ with SD 7.02) than in control $(10.43 \mu \mathrm{mol} / \mathrm{L}$ with SD 2.85). Where, the P-value is $<0.05$, which explains homocysteine level elevated significantly in neurodegenerative diseases (ND). In this study in comparing the serum homocysteine between two groups, an unpaired student t-test was done. Here pvalue was found $0.003(\mathrm{p}<0.05)$ which indicated was a significant correlation between the groups. In this study correlation coefficient (r) of a duration of illness with Hcy is +0.096 . So it has a positive correlation and the strength is also weak; which indicates Hcy increases with the increasing duration of illness in both condition $\mathrm{p}$ values is statistically not significant. The correlation coefficient (r) of $\Delta \mathrm{FS}$ with Hcy is +0.142 ; which indicates Hcy is positively correlated with progression rate $\Delta \mathrm{FS}$, and the correlation is also weak. So there is increasing Hcy level with the increase of progression rate $\triangle \mathrm{FS}$. BMI positively correlated with serum Hcy and age negatively correlated with Hcy.

Table-1: Gender wise distribution between two groups $(\mathrm{N}=84)$

\begin{tabular}{|l|l|l|l|l|l|}
\hline \multirow{2}{*}{ Age (Yrs.) } & \multicolumn{2}{|l|}{ Case(n=42) } & \multicolumn{2}{l|}{ Control(n=42) } & p-Value \\
\cline { 2 - 5 } & n & \% & n & \% & \multirow{1}{10000}{} \\
\hline Male & 33 & 78.57 & 33 & 78.57 & \\
\hline Female & 9 & 21.43 & 9 & 21.43 & \\
\hline Base & 42 & 100.0 & 42 & 100.0 & \\
\hline Male: Female & \multicolumn{3}{|l|}{$3.7: 1$} & & \\
\hline
\end{tabular}

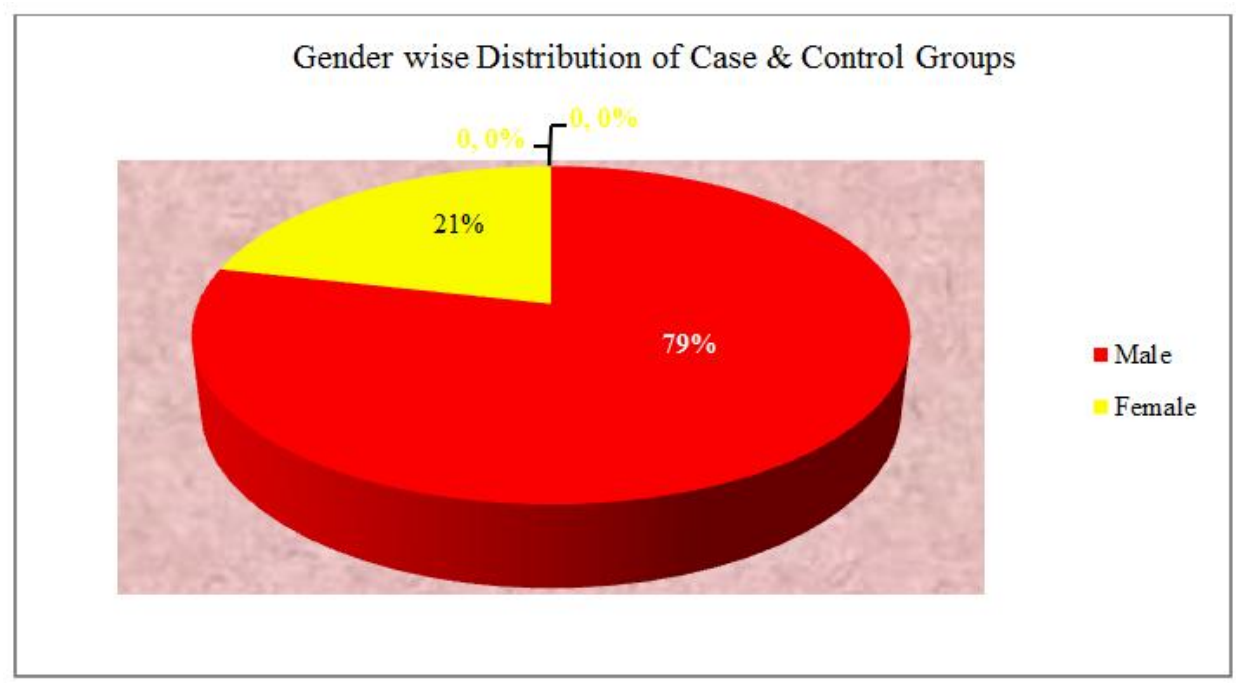

Fig-1: Gender wise Distribution of Case \& Control Groups 
Table-2: Comparison of age between two groups $(\mathrm{N}=84)$

\begin{tabular}{|l|l|l|l|l|l|}
\hline \multirow{2}{*}{ Age(Yrs.) } & \multicolumn{2}{l|}{ Case(n=42) } & \multicolumn{2}{l|}{ Control(n=42) } & \multirow{2}{*}{ p-Value } \\
\cline { 2 - 5 } & $\mathbf{n}$ & $\mathbf{\%}$ & $\mathbf{n}$ & $\mathbf{\%}$ & \multirow{2}{*}{ (05 $^{\text {ns }}$} \\
\hline$<30$ years & 11 & 26.19 & 10 & 23.81 & \\
\hline 30-50 years & 18 & 42.86 & 17 & 40.48 & \\
\hline$>50$ years & 13 & 30.95 & 15 & 35.71 & \\
\hline Base & 42 & 100.0 & 42 & 100.0 & \\
\cline { 1 - 3 } Mean \pm SD & \multicolumn{4}{|l|}{$41.55 \pm 14.9$} & \multicolumn{3}{|l|}{$42.78 \pm 14.87$} & \\
\hline
\end{tabular}

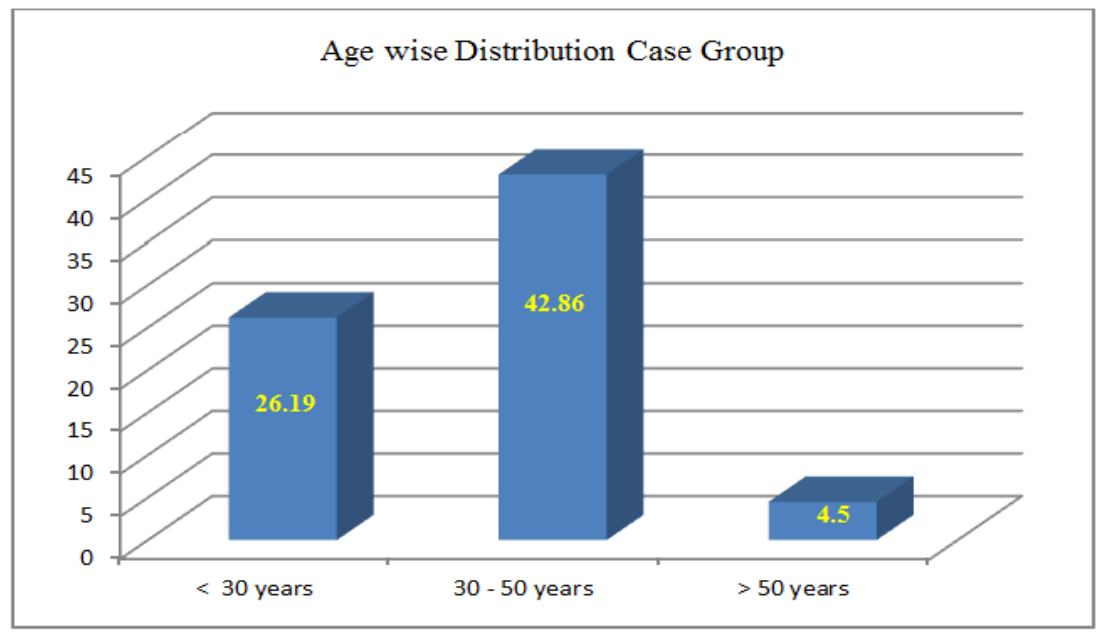

Fig-2: Age wise Distribution of Case Group

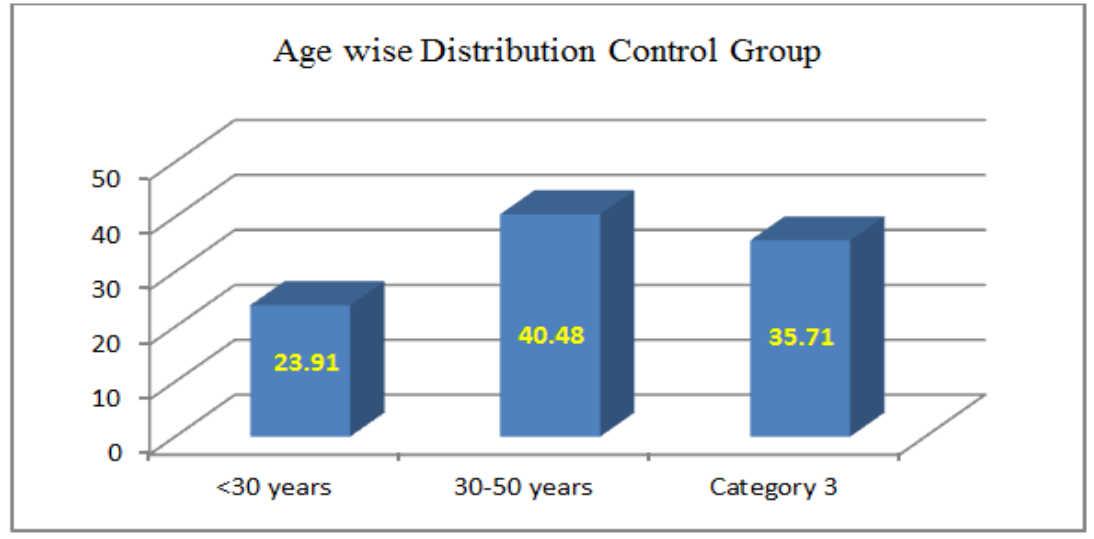

Fig-3: Age wise Distribution of Control Group

Table-3: Comparison of BMI between case \& control groups $(\mathrm{N}=84)$

\begin{tabular}{|l|l|l|l|l|l|}
\hline \multirow{2}{*}{ BMI $(\mathbf{k g} / \mathbf{m} 2)$} & \multicolumn{2}{|l|}{ Case $(\mathbf{n}=\mathbf{4 2})$} & \multicolumn{2}{l|}{ Control $(\mathbf{n}=\mathbf{4 2})$} & \multirow{2}{*}{ p-Value } \\
\cline { 2 - 5 } & $\mathbf{n}$ & $\mathbf{\%}$ & $\mathbf{n}$ & $\mathbf{\%}$ & \\
\hline$<18.5$ & 7 & 16.67 & 4 & 9.52 & \multirow{2}{*}{0.121} \\
\hline $18.5-24.99$ & 35 & 83.33 & 38 & 90.48 & \\
\hline $25.0-29.9$ & 0 & 0 & 0 & 0 & \\
\cline { 1 - 4 } Base & 42 & 100.0 & 42 & 100.0 & \\
\cline { 1 - 3 } Mean \pm SD & \multicolumn{2}{|l|}{$20.26 \pm 2.11$} & \multicolumn{2}{|l|}{$21.35 \pm 2.10$} & \\
\hline
\end{tabular}

Table-4: Disease Patterns of Case groups $(n=42)$

\begin{tabular}{|l|l|l|}
\hline Type of ND Diseases & Frequency (n) & Percentage \%) \\
\hline Alzheimer's disease (AD) & 15 & 36.5 \\
\hline Parkinson's disease(PD) & 11 & 24.5 \\
\hline Amyotrophic lateral sclerosis disease (ALS) & 8 & 19.0 \\
\hline Huntington's disease (HD) & 6 & 15.0 \\
\hline Prion disease & 2 & 5.0 \\
\hline Base & $\mathbf{4 2}$ & $\mathbf{1 0 0 . 0}$ \\
\hline
\end{tabular}




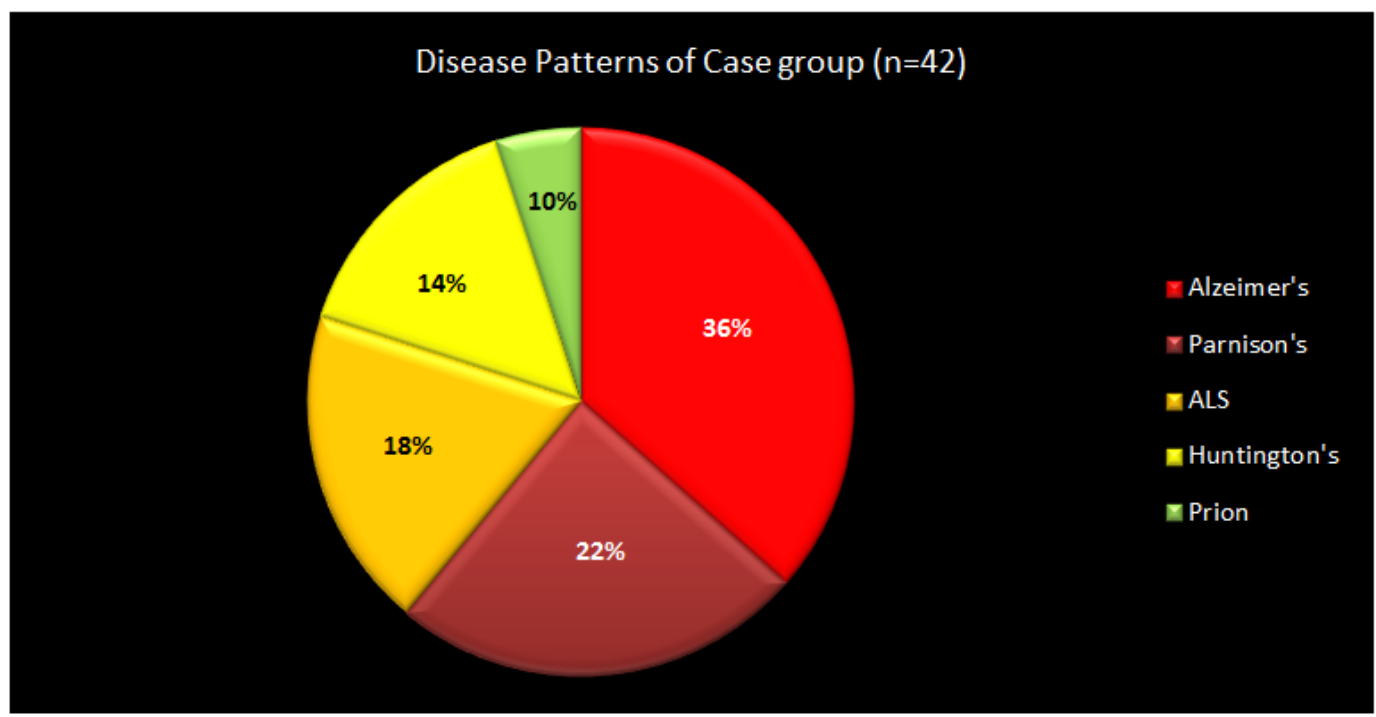

Fig-4: Diseases Patterns of Case Group

Table-5: Complaints of Case Group $(n=42)$

\begin{tabular}{|l|l|l|}
\hline Complaints & $\begin{array}{l}\text { Frequency } \\
(\mathbf{n})\end{array}$ & Percentage (\%) \\
\hline Weakness in all 4 limbs & 31 & 73.81 \\
\hline Weakness in upper limbs & 6 & 14.29 \\
\hline Weakness in lower limbs & 2 & 4.76 \\
\hline Weakness in single limb & 1 & 2.38 \\
\hline Tongue atrophy & 18 & 42.86 \\
\hline Small muscle atrophy & 39 & 92.86 \\
\hline Difficulty in walking & 33 & 78.57 \\
\hline Twitching of muscle & 41 & 97.62 \\
\hline Muscle cramps & 33 & 78.57 \\
\hline Sensory complaints & 1 & 2.38 \\
\hline Speech difficulty & 16 & 38.10 \\
\hline Difficulty in deglutition & 16 & 38.10 \\
\hline Nasal regurgitation & 9 & 21.43 \\
\hline Foot drop & 3 & 7.14 \\
\hline Dropping head and camptocormia & 2 & 4.76 \\
\hline
\end{tabular}

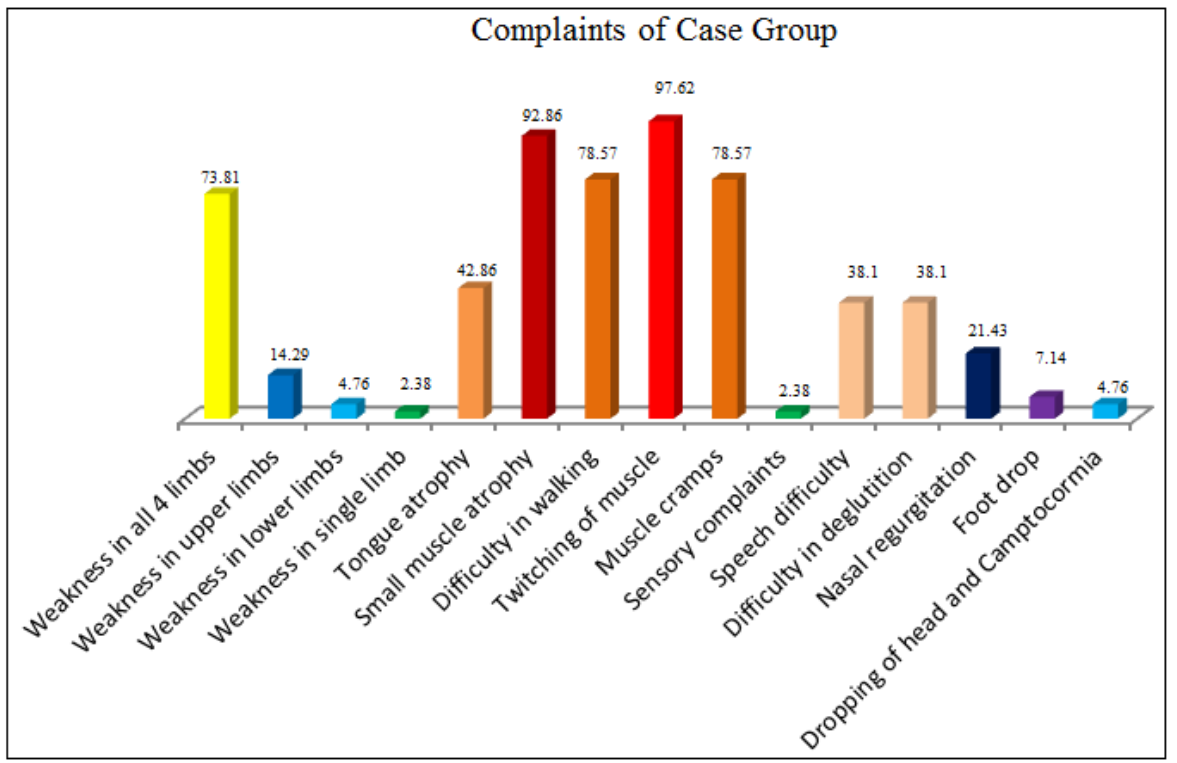

Fig-5: Complaints of Case Group 
Table-6: Duration of illness of Case Group $(n=42)$

\begin{tabular}{|l|l|l|}
\hline Duration & $\begin{array}{l}\text { Frequency } \\
(\mathbf{n})\end{array}$ & Percentage (\%) \\
\hline$<3$ months & 1 & 2.38 \\
\hline $3-6$ months & 12 & 28.58 \\
\hline $7-12$ months & 4 & 9.52 \\
\hline$>12$ months & 25 & 59.52 \\
\hline Base & $\mathbf{4 2}$ & $\mathbf{1 0 0 . 0}$ \\
\hline Mean \pm SD & \multicolumn{2}{|l|}{$14.34 \pm 11.23$} \\
\hline
\end{tabular}

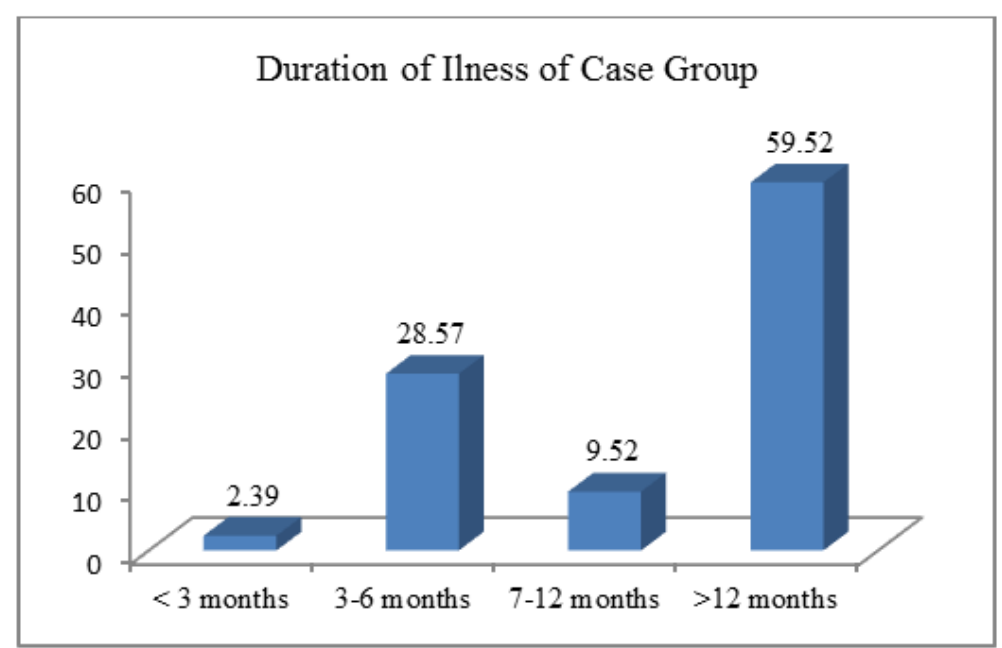

Fig-6: Duration of Illness of Case Group

Table-7: Comparison of serum Homocysteine between two groups ( $n=42)$

\begin{tabular}{|c|c|c|c|c|c|}
\hline \multirow[t]{2}{*}{ Serum Homocysteine(Hcy) } & \multicolumn{2}{|c|}{ Case $(n=42)$} & \multicolumn{2}{|c|}{ Control $(n=42)$} & \multirow{3}{*}{$\begin{array}{l}\text { p-value } \\
0.003\end{array}$} \\
\hline & $\mathbf{n}$ & $\%$ & $\mathbf{n}$ & $\%$ & \\
\hline Elevated Hcy $(>15 \mu \mathrm{mol} / \mathrm{L})$ & 14 & 33.0 & 3 & 7.0 & \\
\hline Normal Hcy $(<15 \mu \mathrm{mol} / \mathrm{L})$ & 28 & 67.0 & 39 & 93.0 & \\
\hline Base & 42 & 100.0 & 42 & 100.0 & \\
\hline Mean \pm SD & \multicolumn{2}{|c|}{$14.06 \pm 7.02$} & \multicolumn{2}{|c|}{$10.43 \pm 2.85$} & \\
\hline
\end{tabular}

Table-8: Correlation of Homocystine with age, disease progression rate, duration of disease and BMI

\begin{tabular}{|l|l|l|}
\hline \multirow{2}{*}{ Variables } & \multicolumn{2}{|l|}{ Serum Homocystine } \\
\cline { 2 - 3 } & Correlation coefficient & p-Value \\
\hline Age (Years) & -0.056 & 0.726 \\
\hline$\Delta$ FS & 0.142 & 0.371 \\
\hline Duration of disease & 0.096 & 0.547 \\
\hline BMI $\left(\mathrm{kg} / \mathrm{m}^{2}\right)$ & 0.162 & 0.336 \\
\hline
\end{tabular}

\section{DiscUSSION}

Homocysteine is a sulfur-containing amino acid derived from methionine, and its plasma concentration is regulated by either re-methylation or transsulfuration pathways. Hyc is converted back into methionine with the help of betainehomocysteinemethyltransferase or methionine synthase and cobalamin (remethylation pathway). It converted to cystathionine by the action of cystathionine betasynthase (CBS) with pyridoxine acting as co-factor. Hyc acts as a precursor and metabolite to S-adenosyl methionine (AdoMet) and S-adenosylhomocysteine (AdoHcy) respectively [17]. The ratio of these 2 is referred to as methylation potential (MP) [20]. In this current study, the respondents were taken from all age groups irrespective of gender and religion. The mean age of the cases was $41.55 \pm 14.9$ years and control was $42.78 \pm 14.87$ years which were quite next to each other. There was no significant difference in age distribution between case and control, ( $p>0.05)$. The minimum age of respondents was 16 years and the maximum age of 78 years. Maximum respondents were between 30-50 years of age group. Alzheimer's disease is characterized by memory loss or mild cognitive impairments (MCI) of normal age with early dementia. Patients of MCI can perform their routine activities smoothly but with some noticeable memory fluctuations or some other sought of cognitive disturbances. MCI could be amnestic and non-amnestic but both of them fail to be diagnosed for 
dementia. MCI could be marked as an outset of dementia due to the fact that patients with MCI are found more prone to dementia than normal cognitive patients. According to the clinical data reports, hyperhomocysteinemia is an independent risk factor in transforming a healthy cognitive person to dementia in both normal elder persons as well as the patient suffering from Alzheimer's disease [25]. Hyperhomocysteinemia is a condition developed in healthy individuals with or without MCI. A co-relation established between learning and hippocampal function and hyperhomocysteinemia suggests that hyperhomocysteinemia degrades cognitive functions in both healthy controls and the case of MCI patients by triggering brain atrophy in patients with MCI [26]. In our study in comparing the serum homocysteine between two groups; an unpaired student t-test was done. Here p-value was found $0.003(\mathrm{p}<0.05)$ which indicated was a significant correlation between the groups. Homocysteine can activate microglia and astrocytes that trigger an inflammatory response which causes neuronal death in patients of Parkinson's disease, the region of the substantianigra $(\mathrm{SN})$ is found to be inflammation and inhibition of this inflammation has proved to be neuroprotective in Parkinson's disease model [27]. The activation of microglia and astrocytes release NO which shows detrimental effects on neurons resulting in neurodegeneration [28]. The NO release can be determined by following MPTP or 6hydroxydopamine (6-OHDA) model in rodents and comparing the neuronal death when only 6-OHDA is administered and when it is co-administered with a NO scavenger [27]. The plasma homocysteine level ranging across $15-100 \mu \mathrm{m}$ gives rise to the condition of mild to moderate hyperhomocysteinemia which has been proven as a risk factor for various neurodegenerative disorders. While the hyperhomocysteinemic condition prevails in the body the raised levels of homocysteine concentration in the brain could be a result of either cellular metabolism within the brain or diffusion and carrier/receptor-mediated transport across the bloodbrain barrier [28]. The aim of this study was to evaluate the serum homocysteine level as an important prognostic marker in neurodegenerative diseases. The correlation coefficient ( $\mathrm{r}$ ) of $\Delta \mathrm{FS}$ with $\mathrm{Hyc}$ is +0.142 ; which indicates $\mathrm{Hyc}$ is positively correlated with progression rate $\Delta \mathrm{FS}$, and the correlation is also weak. So there is increasing Hyc level with the increase of progression rate $\triangle \mathrm{FS}$. BMI positively correlated with serum Hyc and age negatively correlated with Hyc. For this reason, serum homocysteine is being considered as an important prognostic marker in neurodegenerative diseases.

\section{Limitations OF THE STUDY}

This was a single centered study with a small sized sample. So the findings of this study may not reflect the exact scenario of the whole country.

\section{CONCLUSION}

In this study in comparing the Serum Homocysteine between two groups (case and control), an unpaired student t-test was done. Here p-value was found $0.003(\mathrm{p}<0.05)$ which indicated was a significant correlation between the groups. The correlation coefficient (r) of $\triangle \mathrm{FS}$ with $\mathrm{Hyc}$ is +0.142 ; which indicates $\mathrm{Hyc}$ is positively correlated with progression rate $\triangle \mathrm{FS}$, and the correlation is also weak. So there is increasing Hyc level with the increase of progression rate $\triangle F S$. BMI positively correlated with serum Hyc and age negatively correlated with Hyc. For this reason, Serum Homocysteine is being considered as an important prognostic marker in Alzheimer's and other major neurodegenerative diseases. These findings may be helpful for further studies on the same issue.

\section{REFERENCES}

1. Finkelstein JD. The metabolism of homocysteine: pathways and regulation. European Journal of Pediatrics, 1998;157(2):404.

2. Ansari R, Mahta A, Mallack E, Luo JJ. Hyperhomocysteinemia and neurologic disorders: a review. Journal of clinical neurology. 2014 Oct 1;10(4):281-8.

3. Ravaglia G, Forti P, Maioli F, Martelli M, Servadei L, Brunetti N. Homocysteine and folate as risk factors for dementia and Alzheimer disease. Am Journal Clin Nutr, 2005; 82: 636643.

4. Koseoglu E, Karaman Y. Relations between homocysteine, folate and vitamin B12 in vascular dementia and in Alzheimer disease. Clin Biochem, 2007;40:859-863.

5. Mudher A, Lovestone S. Alzheimer's disease-do tauists and bap-tists finally shake hands? Trends Neurosci, 2002;25:22-6.

6. Regland B, Abrahamsson L, Blennow K, Gottfries CG, Wallin A. Vitamin B12 in CSF: reduced $\mathrm{CSF} /$ serum $\mathrm{B} 12$ ratio in dementedmen. ActaNeurolScand 1992; 85:276-81.

7. Bachmann CG, Guth N, Helmschmied K, Armstrong VW, PaulusW, Happe S. Homocysteine in restless legs syndrome. Sleep Med2008; 9:388-92.

8. Levin J, Botzel K, Giese A, Vogeser M, Lorenzl S. Elevated levelsofmethylmalonate and homocysteine in Parkinson's disease, progressivesupranuclear palsy and amyotrophic lateral sclerosis. Dement Geriatr Cogn Disord 2010;29:553-9

9. Camicioli RM, Bouchard TP, Somerville MJ. Homocysteine is notassociated with global motor or cognitive measures in nondementedolder Parkinson's disease patients. Mov Disord, 2009;24:176-82

10. Walker FO. Huntington's disease. Lancet, 2007; 369:218-28. 
11. Boutell JM, Wood JD, Harper PS, Jones AL. Huntingtininteractswithcystathionine betasynthase. Hum Mol Genet, 1998; 7:371-8.

12. Rahman A, Ullah AKM. 'Clinical pattern of Motor neuron disease in Bangladesh'. 2005.

13. Kabiruzzaman M, Islam MR, Miah MBA. Association of serum ferritin with Amyotrophic lateral sclerosis: A case control study. 2012.

14. Kimura F, Fujimura C, Ishida S., Nakajima H, Furutama D, Uehara H, Shinoda K, Sugino M, Hanafusa T. Progression rate of ALSFRS-R at time of diagnosis predicts survival time in ALS. Neurology, 2006;66, 265-267.

15. Finkelstein JD. The metabolism of homocysteine: pathways and regulation. European Journal of Pediatrics, 1998;157(2),404.

16. Ansari R, Mahta A, Mallack E, Luo JJ. Hyperhomocysteinemia and neurologic disorders: a review. Journal of clinical neurology. 2014 Oct $1 ; 10(4): 281-8$.

17. Zhuo JM, Wang H, Praticò D. Is hyperhomocysteinemia an Alzheimer's disease (AD) risk factor, an AD marker, or neither?. Trends in pharmacological sciences. 2011 Sep 1;32(9):562-71.

18. Prusiner SB. The prion diseases. Brain Pathol. 1998; 8(3):499-513.

19. Algaidi SA, Christie LA, Jenkinson AM, Whalley L, Riedel G, Platt B. Long-term homocysteine exposure induces alterations in spatial learning, hippocampal signalling and synaptic plasticity. Experimental neurology. 2006 Jan 1;197(1):8-21.

20. Choe YM, Sohn BK, Choi HJ, Byun MS, Seo EH, Han JY, Kim YK, Yoon EJ, Lee JM, Park J, Woo JI. Association of homocysteine with hippocampal volume independent of cerebral amyloid and vascular burden. Neurobiology of aging. 2014 Jul $1 ; 35(7): 1519-25$.
21. McCully KS. Chemical pathology of homocysteine. IV. Excitotoxicity, oxidative stress, endothelial dysfunction, and inflammation. Annals of Clinical \& Laboratory Science. 2009 Jun 20;39(3):219-32.

22. Knight R, Brazier M, Collins SJ. Human prion diseases: cause, clinical and diagnostic aspects. Contrib Microbiology. 2004; 11:72-97.

23. Tyagi N, Gillespie W, Vacek JC, Sen U, Tyagi SC, Lominadze D. Activation of GABA- A receptor ameliorates homocysteine- induced MMP- 9 activation by ERK pathway. Journal of cellular physiology. $2009 \mathrm{Jul} ; 220(1): 257-66$.

24. Zhang CE, Tian Q, Wei W, Peng JH, Liu GP, Zhou XW, Wang Q, Wang DW, Wang JZ. Homocysteine induces tau phosphorylation by inactivating protein phosphatase $2 \mathrm{~A}$ in rat hippocampus. Neurobiology of aging. 2008 Nov 1;29(11):1654-65.

25. Chen S, Dong Z, Cheng M, Zhao Y, Wang M, Sai $\mathrm{N}$, Wang $\mathrm{X}$, Liu $\mathrm{H}$, Huang $\mathrm{G}$, Zhang $\mathrm{X}$. Homocysteine exaggerates microglia activation and neuroinflammation through microglia localized STAT3 overactivation following ischemic stroke. Journal of neuroinflammation. 2017 Dec $1 ; 14(1): 187$

26. Glass CK, Saijo K, Winner B, Marchetto MC, Gage FH. Mechanisms underlying inflammation in neurodegeneration. Cell. 2010 Mar 19;140(6):91834.

27. Doherty GH. Homocysteine and parkinsons disease: a complex relationship. Journal of Neurological Disorders. 2013 Mar 25:1-9.

28. Kamath AF, Chauhan AK, Kisucka J, Dole VS, Loscalzo J, Handy DE, Wagner DD. Elevated levels of homocysteine compromise blood-brain barrier integrity in mice. Blood. 2006 Jan 15;107(2):591-3. 